\title{
DIAGNOSTICS OF PLASMA JET GENERATED IN WATER/ARGON DC ARC TORCH
}

\author{
Hurba O. ${ }^{a, b, *}$, HlínA M. ${ }^{a}$, HrabovskÝ M. ${ }^{a}$ \\ ${ }^{a}$ Institute of Plasma Physics AS CR, v.v.i., Za Slovankou 1782/3, 18200 Prague 8, Czech Republic \\ ${ }^{b}$ Charles University in Prague, Faculty of Mathematics and Physics, Ke Karlovu 3, 12116 Prague, Czech \\ Republic \\ * ipp@ipp.cas.cz
}

\begin{abstract}
Thermal plasma jet generated by the torch with water/argon stabilized arc was investigated. Plasma torches of this type have been used for plasma spraying, waste treatment and gasification of organic materials. Electric probes, enthalpy probe, and schlieren photography were used for diagnostics of the jet in the region downstream of the torch exit. Information about structure and shape of plasma jet was evaluated from the measured data. Large extent of radial plasma spread and high level of turbulence were found from both the schlieren and the probe diagnostics. Plasma temperature corresponding to measured ion saturation currents was determined using calculated composition of plasma assuming ex-istence of local thermodynamic equilibrium.
\end{abstract}

Keywords: thermal plasma jet, electric probes, schlieren photography, enthalpy probe.

\section{Introduction}

Thermal plasma jets generated in DC arc torches have been utilized in a number of plasma processing technologies like plasma spraying, cutting, waste treatment and gasification of organics. Structure of the jet and conditions in their vicinity, which result from interaction of low density plasma jet with surrounding cold gas atmosphere, are important factors influencing results of plasma processing technologies. A boundary layer instability is produced in the shear flow at the jet boundary [1-3]. Entrainment of cold gas into the plasma jet, which is one of consequences of jet boundary instability, and its effect on jet properties development was described in several papers $[4,5]$. While ambient gas entrainment into plasma jet is well described phenomenon, the second possible mechanism caused by the shear flow instability, an ejection of plasma eddies into surrounding gas, has not been studied so far. We studied the presence of inhomogeneities in the jet vicinity by the schlieren photography, verification of presence of conducting plasma eddies was made by electric probe diagnostics.

\section{Experimental setup}

Steam plasma jet was produced in the hybrid water/gas plasma torch WSP ®H500 (Fig. 1). Argon plasma flows through the cathode noz-zle to the second part of arc chamber where arc column is surrounded by a vortex of wa-ter. The vortex is formed in a chamber with tangential water injection. Water is exhausted out of the chamber in two exhaust gaps. Interaction of the arc column with the water vortex causes evaporation from the inner surface of the vortex. The steam mixes with argon plasma flow-ing from the cathode section. An anode is cre-ated by a rotating copper disc with internal water cooling [6]. The experiments were per-formed at arc current $500 \mathrm{~A}$ and the arc voltage $306 \mathrm{~V}$, Ar flow rate was $22 \mathrm{slm}$.

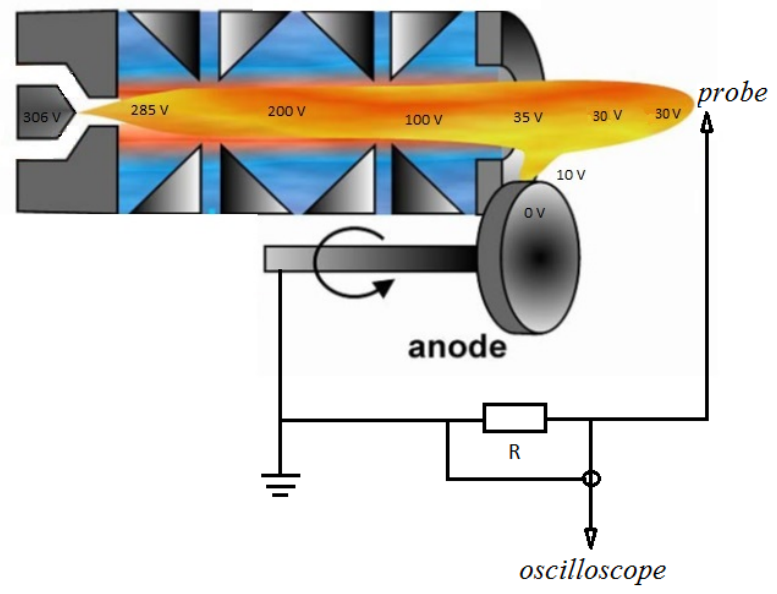

Figure 1. Schematic view of Ar-water stabilised plasma torch WSP BH 500 and scheme of probe circuit

The schlieren photography was made by using pulse Nd laser (pulse width $35 \mathrm{~ns}$ ), schlieren lens with $f=1500 \mathrm{~mm}$ and different radii of circle disc screen positioned in the focus. The diameter of illuminating beam was $50-80 \mathrm{~mm}$, the wavelength $540 \mathrm{~nm}$ (second harmonics) was used. Enthalpy probe system TEKNA ENP-04 was used for measurement of plasma temperature profiles. For an electric probe diagnostic we used molybdenum probe $4 \mathrm{~mm}$ in length and 0.8 in diameter. This probe was connected to the anode through a variable resistor $R$. The probe was passed across the jet at various axial distances from the torch 
nozzle exit, the potential changes of the probe were recorded on an oscilloscope together with a signal of device registering probe position.

\section{Experimental results}

In Fig. 2 the schlieren image of the jet and its vicinity is shown. It may be seen that in the gas around the jet the structures with strong gradients of density are present with well-defined boundaries.

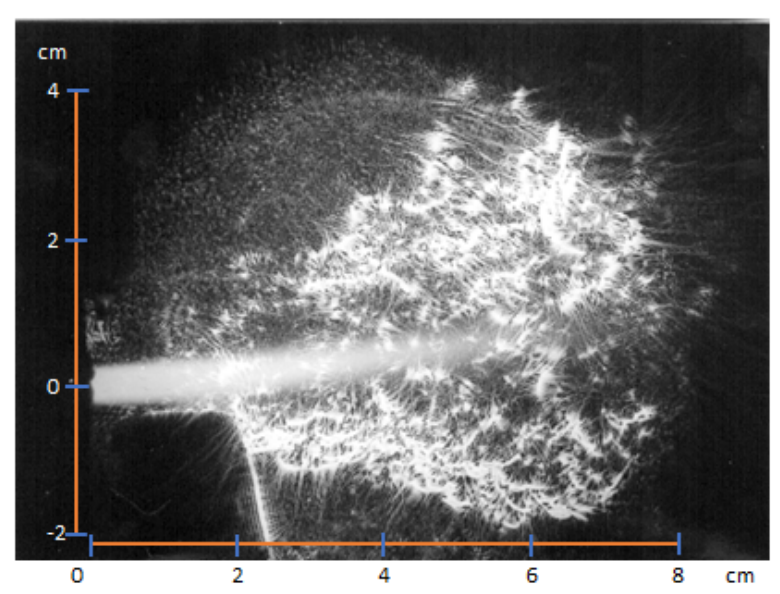

Figure 2. Schlieren photography of plasma jet

The electric probe diagnostics was used to determine electrical conductivity of the region with observed structures. The distribution of potential around plasma jet was meas-ured as well.

The probe potential distribution obtained when electrical probe was moved across the plasma jet at a distance of $50 \mathrm{~mm}$ from plasma torch can be seen in Fig. 3 .

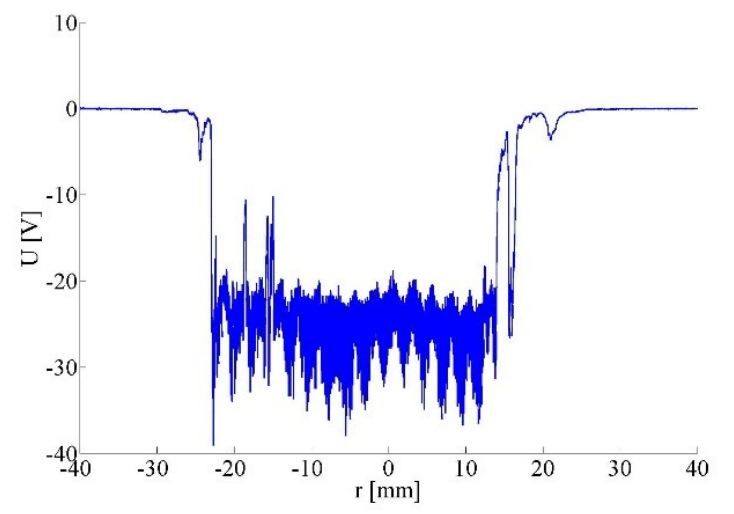

Figure 3. Probe potential measured by moving probe at a distance of $50 \mathrm{~mm}$ from the nozzle exit for biasing resistance $R=1 \mathrm{M} \Omega$

The measurements were made with high bias-ing resistance $R=1 \mathrm{M} \Omega$. The same probe po-tential signal was obtained for higher values of $\mathrm{R}$. Thus, the probe potential was not influ-enced by the probe current, which can be de-termined as $I_{p}=U_{p} / R$, where $U_{p}$ is measured probe potential. Measured potential
$20-35 \mathrm{~V}$ in the plasma jet region (Fig. 3) is given by the voltage drop on an anode attachment, through which arc current flows from the jet to the anode [7].

Probe currents corresponding to measured potential in Fig. 3 were $20-35 \mu \mathrm{A}$. For radial distances from the jet axis larger than about $20 \mathrm{~mm}$, the probe potential falls down rapidly to zero. As probe is positively biased by con-necting to the anode, the probe currents are electron currents. It means that probe poten-tial corresponds approximately to floating plasma potential for positions where probe voltage does not depend on biasing resistance $R$, and falls down when the electron current flowing to probe is lower than $U_{\text {plasma }} / R$. The boundary of conducting area determined from measurements for $R>1 \mathrm{M} \Omega$ is shown in Fig. 4

Axial changes of plasma jet potential obtained by evaluating probe measurements at various axial positions are shown in Fig. 5

A number of measurements were made for various axial positions and various values of biasing resistance R. Fig. 6 presents probe signals measured at a distance $30 \mathrm{~cm}$ from the torch nozzle exit for various values of $R$. While for values of $R$ of order $100 \mathrm{k} \Omega$ the probe signals were the same as for $R=1 \mathrm{M} \Omega$, for lower $\mathrm{R}$ the probe potentials were lower. For $R=1 \mathrm{k} \Omega$ the probe potential was lower than $1 \mathrm{~V}$. It means that probe currents that can flow to the probe are lower than corresponding values of $U_{\text {plasma }} / R$. The probe currents corresponding to the peak values of measured voltage signals in Fig. 7 are in the range $10^{-4}--10^{-3} \mathrm{~A}$ which corresponds to the current densities on the probe tip $10^{2}--10^{1} \mathrm{~A} / \mathrm{m}^{2}$.

We have calculated the electron saturation current density flowing to electrostatic probe from the equation

$$
I_{e s}=n_{e} e\left(\frac{k T}{2 \pi m_{e}}\right)^{1 / 2}
$$

for values of electron density ne calculated from equilibrium composition of steam/argon plasma generated by the torch [8]. The result-ing dependence of electron saturation current density on plasma temperature is shown in Fig. 7.

Our measured probe current densities corre-spond to saturation currents at plasma tem-peratures 4000 $5000 \mathrm{~K}$. As saturation currents are maximum currents that can be collected by the probe, our measured currents correspond to plasma temperatures, which are much higher than temperatures measured at this axial position by the enthalpy probe presented in Fig. 8.

The figure presents temperature profile measured at the position $30 \mathrm{~cm}$ from the torch exit by the enthalpy probe TEKNA. The values of temperatures in Fig. 8 were evaluated from measured plasma enthalpies using calculated thermodynamic properties for composition of gases determined from mass spectra measured by 


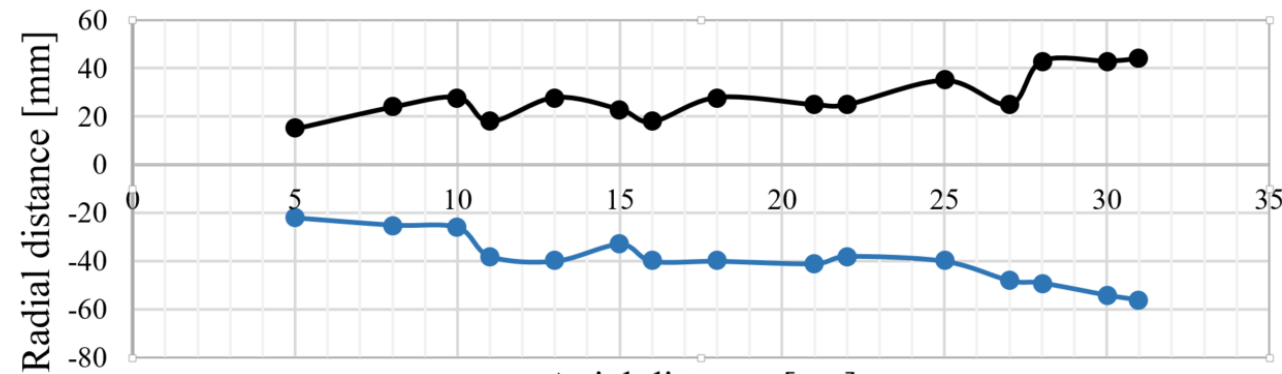

Axial distance $[\mathrm{cm}]$

Figure 4. : Boundaries of the conducting region around plasma jet

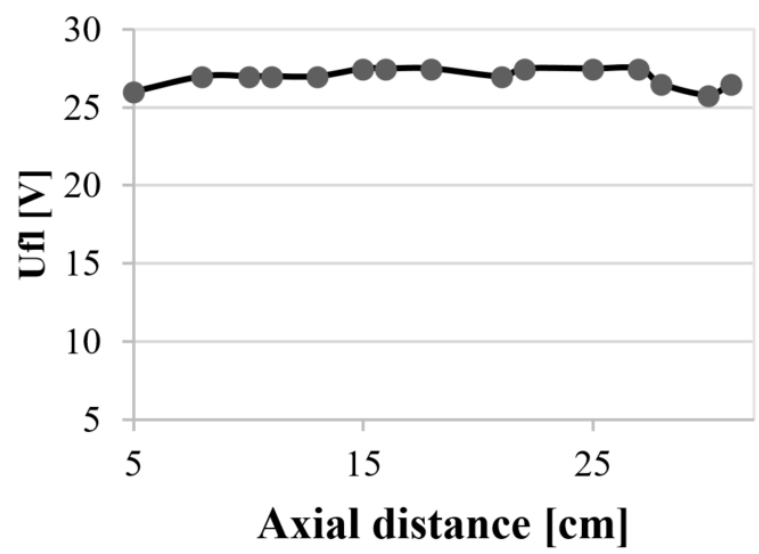

Figure 5. The dependence of plasma jet potential on axial position

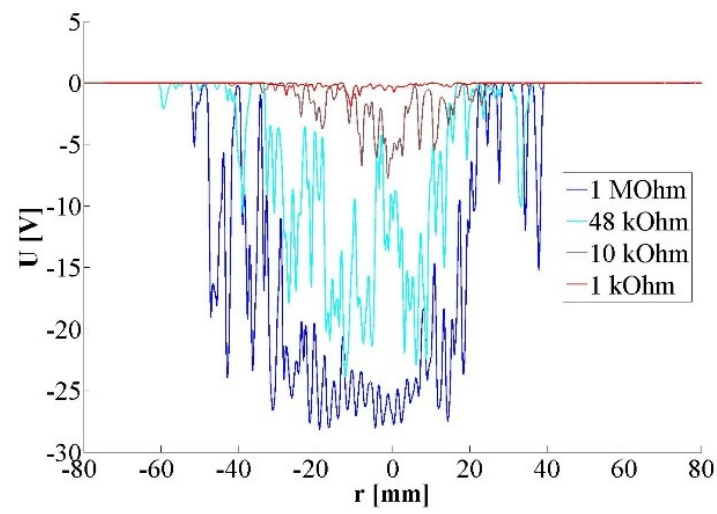

Figure 6. Measured probe potentials for different values of $R$ at the distance $30 \mathrm{~cm}$ from the plasma torch

mass spectrometer, which is part of enthalpy probe system.

It can be seen that temperatures measured by the enthalpy probe are substantially lower then plasma temperatures corresponding to measured probe currents. The difference can be explained by the fact, that enthalpy probe measures averaged temperature of all components of flowing fluid, while electric probe currents are determined by electron density and temperature. We can conclude that the jet is composed of small regions of relatively hot plasma in surround-

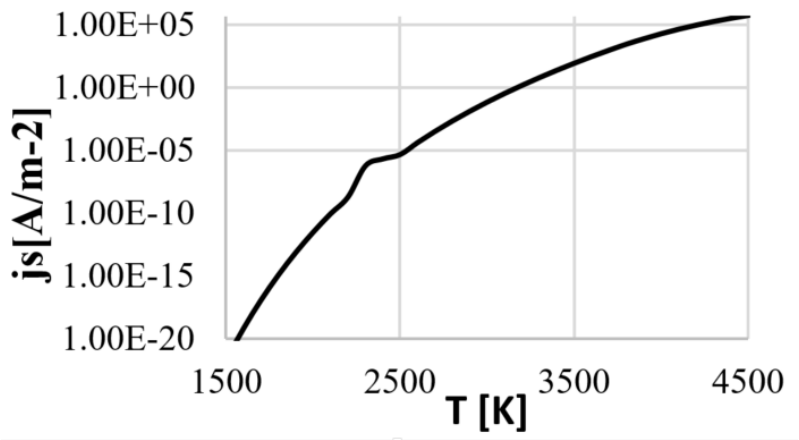

Figure 7. Saturation electron current density in steam/argon plasma (50:50 volume ratio) in dependence on plasma temperature

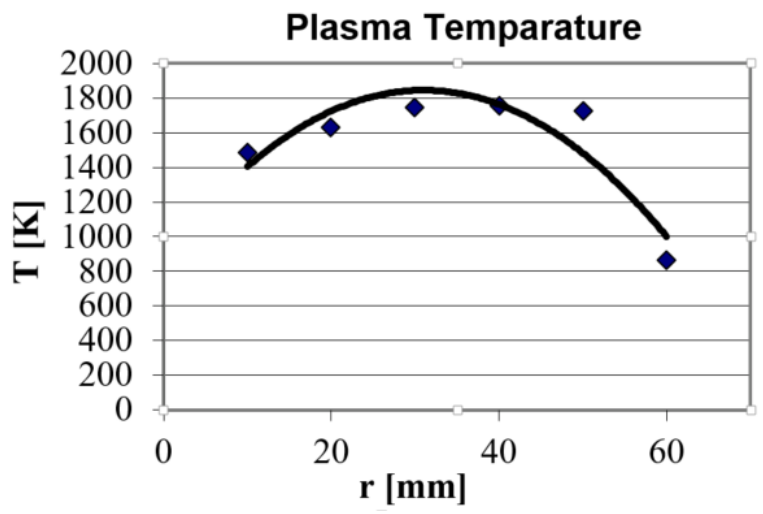

Figure 8. Plasma temperature profile measured by enthalpy probe (the fit "Polynomial 3 degree" has been used for descriptive reasons)

ing colder gas. These two components are not well mixed even at large distances from the torch nozzle exit. This can be seen also on the schlieren image at Fig. 2 Small plasma structures are ejected from plasma jet boundary into surrounding cold gas due to interaction of high velocity plasma stream with steady surrounding cold gas. These plasma structures can survive long time and were detected at large distances from the torch nozzle. 


\section{Conclusions}

Free plasma jet downstream of the plasma torch exit has potential which is determined by voltage drop on the plasma jet attachment to the anode. Surrounding region around the plasma jet is electrically conductive even in large distances from the torch exit nozzle.

Electron currents can flow to the earthed substrate at plasma processing applications and can influence plasma processing result. The conductivity of surrounding of plasma jet is caused by a presence of small plasma structures with temperature substantially higher than average temperature at these positions. These plasma structures were ejected from the plasma jet boundary and survive even in larger distances from the torch.

\section{Acknowledgements}

The authors gratefully acknowledge support of the Grant Agency of CR under the project number GA15-19444S.

\section{References}

[1] Hrabovsky M., Kopecky V. Resonant excitation of boundary layer instability of DC arc plasma jet by current modulation. Plasma Chem. Plama Process., 31:827-838, 2011.
[2] Pentecost C. G., Fincke J. R. Laminar-to-turbulent transitions and entrainment in therml plasma jets. Heat Transfer in Plasma Processing, ASME HTD, 161, 1991.

[3] Kopecky V., Hrabovsky M. Visualization of sructure of boundaty layer between thermal plasma jet and ambient air by moving electric probes. IEEE Trans. on Plasma Science, 33:420-421, 2015.

[4] Strykowski P. J., Russ S. Mixing in plasma and low density jets. Experiments in Fluids, 16:297-307, 1994.

[5] Haggard D. C., Frincke J. R., Swank W. D. Entrainment and demixing in subsonic Argon/Helium thermal plasma jets. In Proc. of 1993 National Thermal Spray Conference, Anaheim, CA: 49-54, 1993.

[6] Konrad M., Kopecky V., Sember V., Brezina V., Hrabovsky M. New plasma spraying torch with combined gas-liquid stabilization of arc. Proceedings of the 15th international symposium on plasma chemistry, vol III, Orleans, 1021-1026, 2001.

[7] Kopecky V., Hlina J., Benes J., Vesely E., Hrabovsky M., Kondrad M. Motion of anode attachment and fluctuation of plasma jet in DC arc plasma torch. Jour. of High Temp. Matrials Processes, 1:167-178, 1997. 